\title{
THE DYNAMICAL EVOLUTION OF TRAPEZIUM SYSTEMS
}

\author{
C. ALLEN and A. POVEDA
}

Instituto de Astronomía, Universidad Nacional Autónoma de México, México, D.F.

\begin{abstract}
To study the dynamical evolution of trapezia, the equations of motion of the member stars in 30 different trapezia were integrated numerically. It was found that two-thirds of the trapezia still remain as such after $10^{6} \mathrm{yr}$. Thus, the dynamical ages of trapezia containing $\mathrm{O}$-stars are only slightly shorter than their nuclear ages. The properties of the binaries formed during the computations are studied. The numerical results are discussed from the observational point of view.
\end{abstract}

\section{Introduction}

Small stellar systems of the trapezium type pose interesting kinematic and dynamical problems, but little attention has, so far, been devoted to them. On the one hand, many workers have studied numerically the dynamical evolution of moderately rich and rich clusters ( 25 to several thousand stars), either by direct integration of the equations of motion, or by various Monte Carlo methods. On the other hand, the analytic solution of the two-body problem is well known and has been used to describe the motions of hierarchical triple or quadruple systems, since these systems are fairly stable over many periods of revolution. Between these extremes, only the unstable three-body problem has received significant attention. It seems therefore necessary, from the purely dynamical point of view, to fill the existing gap by studying the evolution of small stellar clusters ( 4 to 20 stars, say) of the trapezium type (see below for definitions of hierarchical and trapezium-type systems).

There are other reasons as well; observationally, trapezium systems seem to be not uncommon. The best known example is the Orion Trapezium, but already Ambartsumian (1954), in one of the first studies on trapezia, gave a catalogue of 108 such systems. More recently, a search of the Index Catalogue of Double Stars has revealed the existence of many more trapezia (Allen, 1974). Clearly, these samples contain valuable cosmogonical and dynamical information. In fact, as was first pointed out by Wallenquist (1944) and later by Markarian (1950, 1951) and Ambartsumian (1951a, b, 1954) multiple stars of the trapezium type are strongly related to young OB stars, and their space distribution is highly concentrated to the galactic plane.

Finally, several unproven statements about trapezium systems have been propagated in the literature. We cite two examples: (a) that trapezia are in expansion (Parenago, 1953; Ambartsumian, 1954; Sharpless, 1965); (b) that trapezium systems are extremely young (ages of about $10000 \mathrm{yr}$ ) (Parenago, 1953). Previous work (Poveda, 1973; Poveda et.al., 1974) dealt with point (a). One of the aims of the present paper is to clarify point (b).

The way we chose to study the dynamical evolution of trapezium systems is by direct numerical integration of the equations of motion of a sample of 30 different trapezia, over a time of $10^{6} \mathrm{yr}$. In Section 2 the relevant definitions are given; in 
Section 3 we discuss the numerical method and the initial conditions; in Section 4 our results are summarized and discussed; finally, some conclusions are given in Section 5.

\section{Definitions}

According to Ambartsumian (1954), a trapezium-type system, or trapezium, is defined as follows: let a multiple star system (of 3 or more stars) have components $a, b, c, \ldots$; let $a b, a c, b c, \ldots$ denote the distances between the components; if three or more such distances are of the same order of magnitude, then the multiple system is of trapezium type; if no three distances are of the same order of magnitude, then the multiple system is of ordinary type. For the sake of clarity, we shall call these systems hierarchical systems. Two distances are 'of the same order of magnitude', in this context, if their ratio is greater than $1 / 3$ but less than 3 . Usually, only the distances projected on the plane of the sky can be measured. If one uses the projected distances in the definition of a trapezium, the sample of observed trapezia will contain, in addition to true physical trapezia, also optical trapezia and pseudo-trapezia. An optical trapezium is an apparent multiple system, whose components are not connected physically, and appear close together as a result of projection. A pseudo-trapezium is a multiple system of hierarchical type that appears to be of trapezium type due to projection. By means of an elaborate statistical analysis. Ambartsumian (1954) showed that the number of pseudo-trapezia is small among systems of spectral types $\mathrm{O}$ and $\mathrm{B}$. We shall therefore concern ourselves solely with trapezia composed of massive stars.

For a clearer analysis of the dynamical evolution of trapezia, we find convenient to introduce three additional definitions:

If a system contains, among its components, three stars whose mutual distances are of the same order of magnitude, and such that the largest of these distances is less than $40000 \mathrm{AU}$, then the system is a normal trapezium.

If a system contains, among its components, three stars whose mutual distances are of the same order of magnitude, and such that the largest of these distances is greater than $40000 \mathrm{AU}$, then the system is a wide trapezium.

If a system satisfies the definitions of both normal and wide trapezium, then the system is a mixed trapezium.

\section{Initial Conditions and Numerical Method}

Since our aim is to simulate numerically the dynamical behaviour of real trapezia, we must choose initial conditions that correspond as closely as possible with the actual observational material.

The first thing that must be investigated is whether trapezia are systems with positive or negative total energy. In previous work (Poveda, 1973; Poveda et al., 1974) we studied 33 trapezia for which there exist more than four observations (at different times) of position angles and separations; these systems were examined for changes in the separations between their components. The observations cover a long time 
span, generally over $100 \mathrm{yr}$. In none of the 33 trapezia was it possible to establish - not even marginally - the existence of an overall expansion or contraction. In 12 trapezia some change of separation was detected for one or two stars. In the case of the Orion Trapezium only Star E shows a detectable relative motion. If trapezia had positive energies, then their stars should have relative velocities of such magnitudes as to be easily observed over the time intervals considered above. To cite one specific example: for the Orion Trapezium the implied motion would be, in projection, 0.28 " in $130 \mathrm{yr}$, with respect to the center of mass. With exception of Star E, the motions of the other stars are a full order of magnitude smaller.

It was therefore concluded that observational evidence contradicts the idea of trapezia being expanding systems with positive energy. In view of the above results, we have to consider the dynamics of nonexpanding trapezia with negative total energy.

Our initial conditions and parameters are inspired by the observed properties of the Orion Trapezium. We take 6 components with a total mass of $170 M_{\odot}$ and choose our initial conditions as follows:

(i) Each trapezium has two stars of $50 M_{\odot}$, two of $20 M_{\odot}$ and two of $15 M_{\odot}$, contained within a radius of $5000 \mathrm{AU}$. (This radius is our unit of length.)

(ii) Pairs of stars of equal masses were placed symmetrically on the coordinate axes $x, y, z$, at positions \pm 1.0 , respectively; to each of these symmetrical positions, a perturbation of random direction and random magnitude of up to $25 \%$ of the initial radius was superimposed; the perturbed positions are taken as initial positions. This procedure ensures that our systems will satisfy the definition of trapezia.

(iii) Each star was given a velocity of random direction and random magnitude with dispersions of less than $1 \mathrm{~km} \mathrm{~s}^{-1}$; the velocities were then multiplied by an appropriate factor to force the system to satisfy the virial theorem; these virialized velocities are taken as initial velocities. This ensures that our systems will not be expanding or contracting initially.

A total of 30 different systems was integrated for a length of time of $10^{6} \mathrm{yr}$, corresponding approximately to 30 mean crossing times.

The integrations were carried out with a computer programme developed by the Department of Aerospace Engineering and Engineering Mechanics of the University of Texas at Ausţin, which uses a 7th-order Runge-Kutta-Fehlberg technique, and regularizes the equations of motion of the closest pair by means of the KustaanheimoStiefel transformation (Szebehely and Bettis, 1971).

Typically, we had errors in the total energy of the cluster of $\Delta E / E \simeq 3 \times 10^{-8}$ at the end of the run.

\section{Results and Discussion}

\subsection{DyNAMICAL STABILITY}

The dynamical stability shown by the trapezia of our computer simulations is quite surprising. In the past, trapezia were thought to be very unstable dynamical systems, which after a few crossing times, either would evolve to a more stable hierarchical 
configuration, or else would eject stars until only a stable binary was left (Ambartsumian, 1954).

The present study shows, however, that even after $10^{6} \mathrm{yr}$, or about 30 crossing times, out of an original sample of 30 trapezia, 19 systems are still of trapezium type. In other words, the probability that after $10^{6} \mathrm{yr}$ a trapezium remains as such is $63 \%$. Closer examination of these 19 systems shows that they are not identical to the initial trapezia (see Table I). Specifically, only 6 systems have retained all their stars; the remaining

TABLE I

Trapezia that survive after $10^{6} \mathrm{yr}$

\begin{tabular}{lrll}
\hline $\begin{array}{l}\text { Trapezium } \\
\text { No. }\end{array}$ & $\left(r_{i j}\right)_{\max }$ & Member stars & Classification \\
\hline 1 & & & \\
2 & 35.7 & $1,2,3,4,5,6$ & $\mathrm{M}$ \\
3 & 39.3 & $1,3,4,5,6$ & $\mathrm{~W}$ \\
5 & 21.3 & $1,2,3,4$ & $\mathrm{~W}$ \\
7 & 1.1 & $1,2,3,4$ & $\mathrm{~N}$ \\
8 & 56.9 & $1,2,4,6$ & $\mathrm{~W}$ \\
9 & 7.0 & $1,2,4,5,6$ & $\mathrm{~N}$ \\
14 & 25.4 & $1,2,3,4,5$ & $\mathrm{~W}$ \\
15 & 27.1 & $1,2,3,4,5,6$ & $\mathrm{M}$ \\
16 & 69.4 & $1,2,3,4$ & $\mathrm{~W}$ \\
17 & 37.3 & $1,2,3,4,5,6$ & $\mathrm{M}$ \\
18 & 29.5 & $1,2,3,4,5$ & $\mathrm{~W}$ \\
19 & 18.2 & $1,2,3,5,6$ & $\mathrm{~W}$ \\
24 & 21.6 & $1,2,3,4,5,6$ & $\mathrm{M}$ \\
25 & 6.9 & $1,2,3,4,5,6$ & $\mathrm{~N}$ \\
26 & 13.1 & $1,2,4,5,6$ & $\mathrm{M}$ \\
27 & 14.4 & $1,2,3,4$ & $\mathrm{~W}$ \\
28 & 3.3 & $1,2,4,5$ & $\mathrm{~N}$ \\
29 & 39.1 & $1,2,4,6$ & $\mathrm{~W}$ \\
\hline
\end{tabular}

Stars 1 and 2 are of $50 M_{\odot}$, stars 3 and 4 of $20 M_{\odot}$, stars 5 and 6 of $15 M_{\odot}$. $\mathrm{N}$ : normal trapezium; W: wide trapezium; $\mathrm{M}$ : mixed trapezium.

13 ejected one or two stars each. Also, 15 of these 19 trapezia are what we have called wide trapezia. It is important to note that the evolution from initial trapezia to wide trapezia is not a process of expansion; rather, individual stars get thrown successively into weakly bound orbits. We suggest that the bright stars in galactic clusters are wide trapezia that evolved from normal trapezia originally immersed in the substratum of fainter stars that make up the bulk of the cluster. For example, the normal trapezium in Orion, which lies at the center of a rich cluster of infrared stars, may evolve, with the passage of time, into a system resembling the Pleiades, where the brightest stars form a wide trapezium of the type described above.

It is surprising that a total of 9 systems continue to be trapezia of dimensions comparable to their initial dimensions even after $10^{6} \mathrm{yr}$ of dynamical evolution. Put 
in another way, the probability that a normal trapezium continues to be a normal trapezium after $10^{6} \mathrm{yr}$ is $30 \%$.

Let us turn our attention now to the remaining 11 systems, that is, the systems that were no longer trapezia at the end of the computations. Out of these 11 systems 3 dissolved completely, that is, they ejected stars with positive energies, until only a binary was left. In all three cases the binary was composed of the two most massive stars. The remaining 8 cases had evolved, at the end of the computations, into hierarchical systems. Stated differently, the probability that a trapezium dissolves in $10^{6} \mathrm{yr}$ is $10 \%$; the probability that a trapezium becomes a hierarchical system in $10^{6} \mathrm{yr}$ is $27 \%$. The previous results are schematically represented below:

30 trapezia $+10^{6} y r=\left\{\begin{array}{l}19 \text { trapezia }\left\{\begin{array}{c}15 \text { wide trapezia (Nos. } 1,2,3,7,9,14,15,16,17,18,19,25,26,28,29) \\ 9 \text { normal trapezia (Nos. 1, 5, 8, 14, 16, 19, 24, 25, 27) } \\ 5 \text { mixed trapezia (Nos. 1,14,16,19, 25) }\end{array}\right. \\ 3 \text { dissolved systems (Nos. } 4,23,30) \\ 8 \text { hierarchical systems (Nos. } 6,10,11,12,13,20,21,22)\end{array}\right.$

The above results lead us to expect that in an unbiased sample of normal trapezia, the great majority of the systems should be younger than $10^{6} \mathrm{yr}$; in this interval of time no significant evolution of the member stars is to be expected. Therefore, the ratio of $\mathrm{O}$ to $\mathrm{B}$ stars in such a sample of trapezia is just the intrinsic ratio in which these stars are born; in contrast, the ratio of $\mathrm{O}$ to $\mathrm{B}$ stars in the general field or in hierarchical systems should be different, because it is a function also of the ratios of their nuclear ages.

\subsection{COMPARISON WITH OBSERVATIONS}

When attempting to compare our theoretical results with observations, it is important to bear in mind that observational material on trapezia comes from catalogues of double and multiple stars. The inclusion of trapezia in these catalogues will depend on rather artificial selection criteria, like the angular separation of the closest pair (Heintz, 1973). Thus, we would not expect many of the observational counterparts of our wide trapezia to be included in the catalogues. Rather, from the observational point of view, many of these systems should be considered as dissolved, even though, theoretically, the distant stars are still bound to the system. The consideration that trapezia are not isolated systems, and therefore their distant stars are subject to perturbations by galactic tidal forces, passing field stars, etc. lends support to this conclusion. Therefore, our results should be examined anew, from the observational point of view. For this purpose, we introduce an arbitrary, but reasonable, cutoff distance of $40000 \mathrm{AU}$. When a star (or, exceptionally, a close pair) reaches a distance from all the other stars of $40000 \mathrm{AU}$ we stop considering it as a member of the trapezium, even though it may still be weakly bound. Elimination of these stars yields results as summarized below:

\footnotetext{
30 trapezia $+10^{6} y r=\left\{\begin{array}{l}9 \text { normal trapezia (Nos. } 1,5,8,14,16,19,24,25,27) \\ 13 \text { dissolved systems (Nos. } 2,3,4,7,9,15,20,21,22,23,26,28,30) \\ 8 \text { hierarchical systems (Nos. } 6,10,11,12,13,17,18,29)\end{array}\right.$
} 


\subsection{BinARIES}

It was found that, at the end of the computations, 33 binaries had been formed. We want to draw attention to the fact that stars 1 and 2, which are the most massive ones, show a strong tendency to form a binary (see Table II). In fact, in 21 out of 30 trapezia, stars 1 and 2 formed the tightest pair. In all of the trapezia that ejected four stars with positive energies, the left-over binary is composed of stars 1 and 2 . In $77 \%$ of the trapezia that can be considered as dissolved from the observational point of view the same thing is true.

The orbital elements of the 33 binaries are given in Table II. As a group, these binaries are characterized by the following average values of their elements:

$$
\langle a\rangle=0.7000 ; \quad\langle\varepsilon\rangle=0.6581 \text {. }
$$

TABLE II

Orbital elements of binaries

\begin{tabular}{|c|c|c|c|}
\hline $\begin{array}{l}\text { Trapezium } \\
\text { No. }\end{array}$ & $\begin{array}{l}\text { Members } \\
\text { of binary }\end{array}$ & $a$ & $\varepsilon$ \\
\hline 1 & $1-2$ & 0.6078 & 0.2357 \\
\hline 2 & $1-3$ & 0.1354 & 0.9928 \\
\hline 3 & $1-2$ & 0.2468 & 0.2356 \\
\hline 4 & $1-2$ & 0.2293 & 0.6121 \\
\hline 5 & $1-4$ & 0.1969 & 0.6182 \\
\hline 6 & $1-2$ & 0.1301 & 0.5107 \\
\hline 7 & $1-2$ & 0.1758 & 0.9972 \\
\hline \multirow[t]{2}{*}{8} & $1-2$ & 0.1450 & 0.8233 \\
\hline & $4-5$ & 3.8050 & 0.6568 \\
\hline 9 & $2-3$ & 2.0318 & 0.8863 \\
\hline 10 & $1-2$ & 0.3253 & 0.9094 \\
\hline \multirow[t]{2}{*}{11} & $1-2$ & 1.1059 & 0.3953 \\
\hline & $4-6$ & 0.5834 & 0.1958 \\
\hline 12 & $1-2$ & 0.2683 & 0.5211 \\
\hline 13 & $1-2$ & 0.1976 & 0.2228 \\
\hline 14 & $1-2$ & 0.4860 & 0.8403 \\
\hline 15 & $1-2$ & 0.1966 & 0.9551 \\
\hline 16 & $2-6$ & 0.7765 & 0.7620 \\
\hline 17 & $1-2$ & 0.3313 & 0.7710 \\
\hline 18 & $1-2$ & 0.3041 & 0.7131 \\
\hline 19 & $1-4$ & 3.6753 & 0.9523 \\
\hline \multirow[t]{2}{*}{20} & $1-2$ & 0.1894 & 0.6273 \\
\hline & $4-6$ & 3.8602 & 0.5767 \\
\hline 21 & $1-2$ & 0.2660 & 0.2140 \\
\hline 22 & $1-2$ & 0.2586 & 0.3024 \\
\hline 23 & $1-2$ & 0.2644 & 0.9213 \\
\hline 24 & $1-2$ & 0.4929 & 0.9463 \\
\hline 25 & $1-2$ & 0.3271 & 0.7992 \\
\hline 26 & $1-2$ & 0.2877 & 0.9616 \\
\hline 27 & $2-5$ & 0.0620 & 0.3457 \\
\hline 28 & $2-4$ & 0.1380 & 0.9413 \\
\hline 29 & $1-2$ & 0.8114 & 0.6371 \\
\hline 30 & $1-2$ & 0.1893 & 0.6377 \\
\hline
\end{tabular}


It is to be noticed that the value of the mean eccentricity of the systems agrees very closely with $2 / 3$, which is the value of the mean eccentricity expected for an ensemble of binaries whose distribution function in phase space is a function only of the energy of the pair (Ambartsumian, 1937). Furthermore, the distribution of eccentricities of our pairs follows approximately the Jeans-Ambartsumian law $N(\varepsilon) \mathrm{d} \varepsilon=2 \varepsilon \mathrm{d} \varepsilon$. No correlation was found between the periods and eccentricities of the 33 binaries. This result is in agreement with the observed properties of visual binaries (Heintz, 1969).

\section{Conclusions}

Trapezium-type systems are much more stable than previously thought; it was found that after $10^{6} \mathrm{yr}$, or about 30 crossing times, $63 \%$ of the trapezia still remain as such. Therefore, the dynamical lifetimes of trapezia containing $O$ stars are not much smaller than their nuclear ages.

In the same interval of time, only $10 \%$ of the trapezia dissolve, leaving behind a binary star; about $27 \%$ of the trapezia evolve into ordinary hierarchical systems.

A very strong tendency was shown by the two massive stars to join into a bound pair.

The distribution of the eccentricities of the binaries resembles the Jeans-Ambartsumian law; the mean eccentricity agrees with the value predicted by this law.

\section{Acknowledgements}

We gratefully acknowledge that the computations were done at the Centro de Servicios de Cómputo, Universidad Nacional Autónoma de México. It is a pleasure to thank Dr Sebastian von Hoerner for suggesting an algorithm to determine the state of hierarchization of a multiple system. We also thank Mrs L. Parrau and Mr M. Tapia for computational assistance.

\section{References}

Allen, C.: 1974 , in preparation.

Ambartsumian, V. A.: 1937, Astron. J. U.S.S.R. 14, 207.

Ambartsumian, V. A.: 1951a, Publ. Acad. Sci. Armenia 13, 97.

Ambartsumian, V. A.: 1951b, Publ. Acad. Sci. Armenia 13, 129.

Ambartsumian, V. A.: 1954, Contr. Obs. Byurakan 15, 3.

Heintz, W. D.: 1969, J. Roy. Astron. Soc. Canada 63, 275.

Heintz, W. D.: 1973, cited in J. Roy. Astron. Soc. Canada 67, 67.

Markarian, B. E.: 1950, Bull. Obs. Byurakan 5, 3.

Markarian, B. E.: 1951, Bull. Obs. Byurakan 9, 3.

Parenago, P. P.: 1953, Astron. J. U.S.S.R. 30, 249.

Poveda, A.: 1973, reported by W. D. Heintz in J. Roy. Astron. Soc. Canada 67, 65.

Poveda, A., Allen, C., and Worley, C.: 1974, in preparation.

Sharpless, S.: 1965, Vistas in Astronomy 8, 127.

Szebehely, V. and Bettis, D.: 1971, Astrophys. Space Sci. 13, 365.

Wallenquist, A.: 1944, Uppsala Ann. 1, No. 5. 


\section{DISCUSSION}

R. H. Miller: If an observer looked at one of your trapezium systems at an age of $10^{6} \mathrm{yr}$, could he conclude that the age was only $10^{4} \mathrm{yr}$ ?

A. Poveda: On projection our clusters will not show a general expansion, but the random motions of the stars. Therefore, an expansion age of $10^{4} \mathrm{yr}$ could not be inferred from our calculations, as it cannot be inferred from observations either.

S. J. Aarseth: I would like to mention a new regularization method developed by Zare and myself for the general three-body problem. All motions can be studied, except the case of triple collisions, and perturbations from other bodies can be included. Furthermore, Heggie has developed a global regularization method for the general $N$-body problem in which all binary collisions can be regularized. 